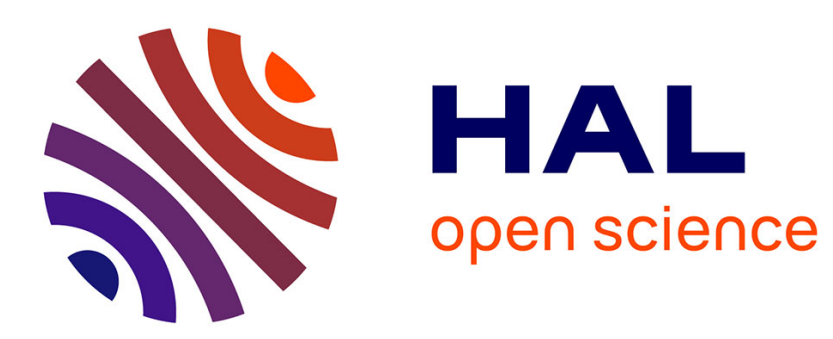

\title{
Tire/Road Contact Condition Identification Using Algebraic Numerical Differentiation
}

\author{
Lei Yu, Jean-Pierre Barbot, Thierry Floquet
}

\section{To cite this version:}

Lei Yu, Jean-Pierre Barbot, Thierry Floquet. Tire/Road Contact Condition Identification Using Algebraic Numerical Differentiation. IFAC Symposium on System Identification, SYSID, 2009, SaintMalo, France. inria-00374418

\section{HAL Id: inria-00374418 https://hal.inria.fr/inria-00374418}

Submitted on 8 Apr 2009

HAL is a multi-disciplinary open access archive for the deposit and dissemination of scientific research documents, whether they are published or not. The documents may come from teaching and research institutions in France or abroad, or from public or private research centers.
L'archive ouverte pluridisciplinaire HAL, est destinée au dépôt et à la diffusion de documents scientifiques de niveau recherche, publiés ou non, émanant des établissements d'enseignement et de recherche français ou étrangers, des laboratoires publics ou privés. 


\title{
Tire/Road Contact Condition Identification Using Algebraic Numerical Differentiation
}

\author{
Lei $\mathrm{YU}^{*, * *, * * * *}$ Jean-Pierre BARBOT ${ }^{* *, * * * *}$ \\ Thierry Floquet ${ }^{* * *, * * * *}$ \\ * Signal Processing Laboratory, Electronic and Information School, \\ Wuhan University, China. \\ ** ECS-EA 3649 Equipe Commande des Systèmes, France. \\ *** LAGIS (CNRS, UMR 8146), École Centrale de Lille, BP 48, Cité \\ Scientifique, 59650 Villeneuve d'Ascq, France \\ **** Équipe Projet ALIEN, INRIA Lille-Nord Europe, France.
}

\begin{abstract}
In this paper, a realistic simulation model for Wheeled Mobile Robot (WMR) is given by a dynamical system that switches between three models corresponding to three different tire/road contact conditions: ideal condition, skidding condition and slipping condition. Then, an algebraic based numerical identification for the discrete state (tire/road contact condition) of this switching system is proposed. Finally, specific estimators for the uncertain parameters encountered in the identification scheme are given.
\end{abstract}

Keywords: Mobile robot, tire/road contact estimation, algebraic numerical differentiation.

\section{INTRODUCTION}

For a Wheeled Mobile Robot (WMR), driven following a curve with large curvature and with imperfect tire/road contact condition, there may exist a sliding phenomenon, which is composed by slipping and skidding effects. Considering the pure rolling case as an ideal case for the tire/road contact condition, the slipping and skidding can be considered as fault occurrences. Identification of the tire/road contact condition is also very important for the control of mobile robot, especially for the open loop control system.

The control problem for a WMR has been intensively studied. Most of the papers assume that the tire/road condition is perfect, which is not the case in reality [3], [5], [6], [15]. Recently, the modeling of the sliding phenomenon has attracted a lot of interest. In [2], a dynamic model, called lumped friction model, was introduced to simulate the tire/road contact friction and then an observer was built to estimate the friction only using the wheel angular velocity. However, this model can only be used in order to detect the slipping effects but not the skidding effects. In [11], a complete model with kinematic and dynamic formulas was built under a singular perturbation approach for a WMR that does not satisfy the ideal conditions. Considering that the model used in [11] is too complicated to handle with, in [8] the model was decoupled into three submodels, corresponding to the ideal, skidding and slipping cases. In [13], the slipping effects and skidding effects were considered due to the deformation of the tire and was treated as perturbations on the lateral and longitude dynamics, respectively, and finally kinematic models for four kinds of configurations of the WMR were proposed. In [18], a new tire traction/braking model was proposed in order to detect the "immobilization" (slipping condition) using an extended Kalman filter framework.

In this paper, the model proposed in [11] is used. In order to simplify the problem, the complete model is decoupled into three parts according to [8]. Then, the WMR can be represented as a switching system. Afterwards, considering that the position and the orientation of the robot, $(x, y, \theta)$, and the input torques are measured, an identification scheme for the tire/road contact condition is built using algebraic numerical differentiation, which is a convenient method to estimate the time derivatives of some noisy measurements [1], [9], [10], [14], [17]. In practice, some of the parameters in the WMR model may vary because of exogenous and unknown effects, such as loading or unloading of the mobile robot, irregularity of the road surface, coefficients of the tire materials etc. As a matter of fact, it is also necessary to estimate the parameters. In [16], a higher order sliding mode approach was used to identify some vehicle parameters.

This paper is organized as follows. In Section 2, a dynamical system for the WMR that switches between three models, corresponding to three different tire/road contact conditions, is derived. In Section 3, an estimator for the discrete states (that gives the information on the tire/road contact condition) is designed using algebraic numerical differentiation. In Section 4, some uncertain parameters of the switching system are estimated. Simulation results are given in Section 5.

\section{MODEL DESIGN}

\subsection{Model for the WMR without Nonholonomic Constraints}

In [11], a model for a unicycle type mobile robot (see Fig. 1) was proposed under a singular perturbation ap- 
proach when the nonholonomic constraint is not satisfied.

\section{Assumptions}

(1) The ground is flat, thus, the potential energy is zero.

(2) The unicycle mass is located at the center of the two driving wheels. (Point $N$ in Fig. 1.)

(3) The cornering (resp. longitudinal) stiffness coefficient is the same for both driving wheels.

(4) The velocities of both driving wheels at their center in the expression of the interaction forces are taken to be identical, and more precisely, equal to their average: $\left\|V_{O_{1}}\right\| \approx\left\|V_{O_{2}}\right\|=\sqrt{\dot{x}^{2}+\dot{y}^{2}+l^{2} \dot{\theta}^{2}}$

where $x, y$ are the coordinates of point $N$ in Fig. 1 , and $\theta$ is the orientation angle.

Then, the dynamical system can be described as follows:

$$
\left(\begin{array}{c}
\dot{\rho} \\
\dot{\eta} \\
\epsilon \dot{\mu}
\end{array}\right)=g_{0}(\rho, \eta, \mu)+g_{1} u_{1}+g_{2} u_{2}
$$

where $\rho=(x, y, l \theta)^{T} \in \Re^{3}$ with $l$ the length between the two fixed wheels. $\eta=\left(\eta_{1}, \eta_{2}\right)^{T} \in \Re^{2}$ is a velocity vector. $u=\left(u_{1}, u_{2}\right)^{T} \in \Re^{2}$ represents the torques on each driving wheel and $\mu=\left(\mu_{1}, \mu_{2}, \mu_{3}\right)^{T} \in \Re^{3}$ where $\mu_{1}$ is the velocity perturbation along the lateral movement and $\mu_{2}, \mu_{3}$ represent the velocity perturbation along the longitudinal movement of each driving wheel. One also has:

$$
\begin{aligned}
& g_{0}=\left[\begin{array}{c}
c(\theta) \epsilon \mu_{1}-s(\theta)\left(\eta_{1}+\epsilon \mu_{2}-\epsilon \mu_{3}\right) \\
s(\theta) \epsilon \mu_{1}+c(\theta)\left(\eta_{1}+\epsilon \mu_{2}-\epsilon \mu_{3}\right) \\
\eta_{2}+\epsilon \mu_{2}+\epsilon \mu_{3} \\
-\eta_{1}-\eta_{2}+\epsilon \mu_{2} \\
-\eta_{1}+\eta_{2}-\epsilon \mu_{3} \\
g_{0}^{6} \\
g_{0}^{7} \\
g_{0}^{8} \\
g_{0}^{9}(q, \eta, \mu, \epsilon) \\
g_{0}^{10}(q, \eta, \mu, \epsilon)
\end{array}\right] \\
& g_{1}=\frac{r^{2}}{3 I_{\phi}}\left(\begin{array}{lllllllllll}
0 & 0 & 0 & 0 & 0 & -1 & -1 & 0 & 1 & 0
\end{array}\right)^{T} \\
& g_{2}=\frac{r^{2}}{3 I_{\phi}}\left(\begin{array}{lllllllllll}
0 & 0 & 0 & 0 & 0 & -1 & 1 & 0 & 0 & -1
\end{array}\right)^{T}
\end{aligned}
$$

and

$$
\begin{aligned}
g_{0}^{6}= & \left(\frac{r^{2} G_{y}}{I_{\phi} v}-\frac{G_{y}}{m v}\right)\left(\mu_{2}-\mu_{3}\right) \\
& -\frac{1}{3 l} \epsilon \mu_{1}\left(\eta_{2}+\epsilon \mu_{2}+\epsilon \mu_{3}\right) \\
g_{0}^{7}= & \left(\frac{r^{2} G_{y}}{I_{\phi} v}-\frac{l^{2} G_{y}}{I_{\theta} v}\right)\left(\mu_{2}+\mu_{3}\right) \\
g_{0}^{8}= & -\frac{2 G_{x}}{m v} \mu_{1}+\frac{1}{l}\left(\eta_{1}+\epsilon \mu_{2}-\epsilon \mu_{3}\right)\left(\eta_{2}+\epsilon \mu_{2}+\epsilon \mu_{3}\right) \\
g_{0}^{9}= & -\frac{G_{y}}{m v}\left(\mu_{2}-\mu_{3}\right)-\frac{r^{2} G_{y}}{I_{\phi} v} \mu_{2}-\frac{l^{2} G_{y}}{I_{\theta} v}\left(\mu_{2}+\mu_{3}\right) \\
& -\frac{1}{3 l} \epsilon \mu_{1}\left(\eta_{2}+\epsilon \mu_{2}+\epsilon \mu_{3}\right) \\
g_{0}^{10}= & \frac{G_{y}}{m v}\left(\mu_{2}-\mu_{3}\right)-\frac{r^{2} G_{y}}{I_{\phi} v} \mu_{3}-\frac{l^{2} G_{y}}{I_{\theta} v}\left(\mu_{2}+\mu_{3}\right) \\
& +\frac{1}{3 l} \epsilon \mu_{1}\left(\eta_{2}+\epsilon \mu_{2}+\epsilon \mu_{3}\right)
\end{aligned}
$$

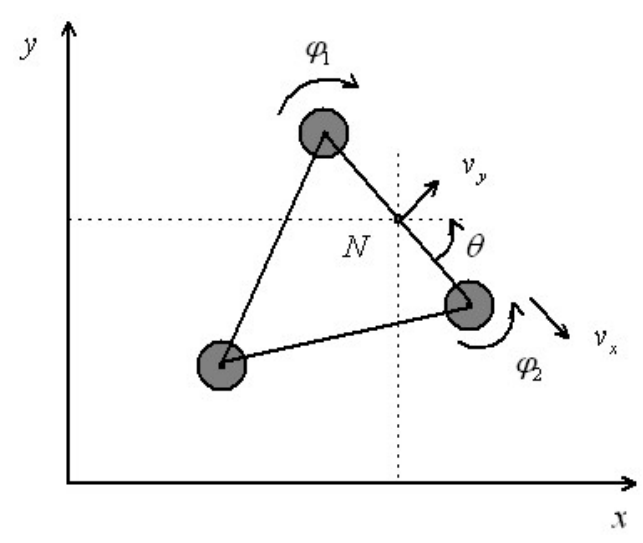

Fig. 1. The Wheeled Mobile Robot

where $m$ is the total mass of the unicycle, $v$ is the average velocity of the point $N$ in Fig. $1, G_{x}, G_{y}$ are the normalized cornering and longitudinal stiffness coefficients and $\epsilon=$ $\min \left\{\frac{1}{G_{x}}, \frac{1}{G_{y}}\right\} . I_{\theta}$ and $I_{\phi}$ are the vertical platform and the wheels moments of inertia, respectively, and $r$ is the wheel radius.

Assuming that the planes of the wheels are vertical to the ground, the contact between the tire and the road can be divided into three cases: an ideal condition (which happens when the WMR is moving with a constant velocity), a skidding condition (which happens when yawing) and a slipping condition (which happens when accelerating or braking). Hereafter, the three cases are discussed and the corresponding models are given.

For decoupling reasons, the following transformation is introduced:

$$
\begin{aligned}
& v_{x}=\epsilon \mu_{1} \\
& v_{y}=\eta_{1}+\epsilon \mu_{2}-\epsilon \mu_{3} \\
& v_{\theta}=\eta_{2}+\epsilon \mu_{2}+\epsilon \mu_{3} \\
& \sigma_{1}=\epsilon\left(\mu_{2}+\mu_{3}\right) \\
& \sigma_{2}=\epsilon\left(\mu_{2}-\mu_{3}\right)
\end{aligned}
$$

where $v_{x}, v_{y}, v_{\theta}$ represents the lateral velocity, the longitudinal velocity, and the angular velocity, respectively.

\subsection{Ideal Condition}

In this case, the velocity of the contact point between the tire and the road is zero, i.e. the nonholonomic constraints exist and there is no slip friction. Thus $\mu_{1}=\mu_{2}=\mu_{3}=0$. Under this condition, the system (1) can be simplified as follows:

$$
\left(\begin{array}{c}
\dot{x} \\
\dot{y} \\
\dot{l \theta} \\
\dot{v}_{x} \\
\dot{v}_{y} \\
\dot{v}_{\theta} \\
\dot{\sigma}_{1} \\
\dot{\sigma}_{2}
\end{array}\right)=\left(\begin{array}{c}
-v_{y} \sin \theta \\
v_{y} \cos \theta \\
v_{\theta} \\
0 \\
-\frac{r^{2}}{3 I_{\phi}}\left(u_{1}+u_{2}\right) \\
-\frac{r^{2}}{3 I_{\phi}}\left(u_{1}-u_{2}\right) \\
0 \\
0
\end{array}\right)
$$




\subsection{Skidding Condition}

In this case, the nonholonomic constraint is violated, since the lateral velocity of the robot is not equal to zero. This means that the perturbation $\mu_{1} \neq 0$, even if $\mu_{2}=\mu_{3}=0$. Then, the system (1) is given by:

$$
\left(\begin{array}{c}
\dot{x} \\
\dot{y} \\
l \dot{\theta} \\
\dot{v}_{x} \\
\dot{v}_{y} \\
\dot{v}_{\theta} \\
\dot{\sigma}_{1} \\
\dot{\sigma}_{2}
\end{array}\right)=\left(\begin{array}{c}
v_{x} \cos \theta-v_{y} \sin \theta \\
v_{x} \sin \theta+v_{y} \cos \theta \\
v_{\theta} \\
-\frac{2 G_{x}}{\epsilon m v} v_{x}+\frac{v_{y} v_{\theta}}{l} \\
-\frac{v_{x} v_{\theta}}{3 l}-\frac{r^{2}}{3 I_{\phi}}\left(u_{1}+u_{2}\right) \\
-\frac{r^{2}}{3 I_{\phi}}\left(u_{1}-u_{2}\right) \\
0 \\
0
\end{array}\right)
$$

\subsection{Slipping Condition}

In this case, the nonholonomic constraint is satisfied, but the velocity of the contact point between the tire and the road is different from zero, i.e. the perturbations satisfy $\mu_{1}=0$ and $\mu_{2} \neq 0, \mu_{3} \neq 0$. Then, the system (1) can be written as follows:

$$
\left(\begin{array}{c}
\dot{x} \\
\dot{y} \\
l \dot{\theta} \\
\dot{v}_{x} \\
\dot{v}_{y} \\
\dot{v}_{\theta} \\
\dot{\sigma}_{1} \\
\dot{\sigma}_{2}
\end{array}\right)=\left(\begin{array}{c}
-v_{y} \sin \theta \\
v_{y} \cos \theta \\
v_{\theta} \\
0 \\
3 G_{y} \sigma_{2} \\
-\frac{\epsilon m v}{\epsilon l^{2} G_{y}} \\
-\left(\frac{r^{2} G_{y}}{\epsilon I_{\theta} v}+\frac{2 l^{2} G_{y}}{\epsilon I_{\theta} v}\right) \sigma_{1}+\frac{r^{2}}{3 I_{\phi}}\left(u_{1}-u_{2}\right) \\
-\left(\frac{r^{2} G_{y}}{\epsilon I_{\phi} v}+\frac{2 G_{y}}{\epsilon m v}\right) \sigma_{2}+\frac{r^{2}}{3 I_{\phi}}\left(u_{1}+u_{2}\right)
\end{array}\right)
$$

\section{DISCRETE STATE IDENTIFICATION}

\subsection{Switching System for WMR}

In the previous section, the 3 cases for the tire/road contact condition were discussed and it was assumed that the different kind of contacts could not occur simultaneously. However, both slipping and skidding may occur at the same time, since the surface of the road can give rise to both phenomenons. In this paper, it is assumed that slipping and skidding are separated but simultaneous occurrence of both phenomenons will be considered in a future work. Here, the problem is considered as an hybrid problem with 3 discrete states, i.e. $q=0$ for the ideal case, $q=1$ for the skidding case and $q=2$ for the slipping case. Then, the objective is to design an observer in order to estimate the discrete state of the hybrid systems, and thus to know whether the WMR is sliding or not.

Under the assumptions, the WMR can be described by a switching system, denoted by $S$ :

$$
S:\left\{\begin{array}{c}
\dot{\xi}=f_{q}(\xi, u), \quad \xi\left(t_{0}, 0\right)=\xi_{0} \\
s=(x, y, \theta)^{T}
\end{array}\right.
$$

where $\xi=\left(x, y, \theta, v_{x}, v_{y}, v_{\theta}, \sigma_{1}, \sigma_{2}\right)^{T} \in \Re^{8}$ is the continuous states, $q \in Q=\{0,1,2\}$ is the discrete state and is determined by the tire/road contact condition, $u \in \Re^{2}$ is the inputs, $f_{q} \in \Re^{8}$ corresponds to each dynamical function and $s \in \Re^{3}$ is the output vector. $q=0,1,2$ represents the ideal case (3), the skidding case (4) and the slipping case (5), respectively. The state jumps are defined as follows:

$$
\begin{aligned}
& \xi\left(t_{k}, k\right)=\left(\begin{array}{cccc}
I_{3 \times 3} & 0 & 0 & 0 \\
0 & 0 & 0 & 0 \\
0 & 0 & I_{2 \times 2} & 0 \\
0 & 0 & 0 & 0_{2 \times 2}
\end{array}\right) \cdot \xi\left(t_{k-1}^{\prime}, k-1\right) \quad q_{k}=0 \\
& \xi\left(t_{k}, k\right)=\left(\begin{array}{cccc}
I_{3 \times 3} & 0 & 0 & 0 \\
0 & 1 & 0 & 0 \\
0 & 0 & I_{2 \times 2} & 0 \\
0 & 0 & 0 & 0_{2 \times 2}
\end{array}\right) \cdot \xi\left(t_{k-1}^{\prime}, k-1\right) \quad q_{k}=1 \\
& \xi\left(t_{k}, k\right)=\left(\begin{array}{cccc}
I_{3 \times 3} & 0 & 0 & 0 \\
0 & 0 & 0 & 0 \\
0 & 0 & I_{2 \times 2} & 0 \\
0 & 0 & 0 & I_{2 \times 2}
\end{array}\right) \cdot \xi\left(t_{k-1}^{\prime}, k-1\right) \quad q_{k}=2
\end{aligned}
$$

where $\left[t_{k}, t_{k}^{\prime}\right)$ is the time interval of the discrete state $q_{k}$, $I_{i \times i}$ is the identity matrix with $i \times i$ dimension.

\subsection{Observability of each subsystems}

Let us recall the definition of the observability of a nonlinear system in the sense given in [7].

Lemma 1. Choose a subset $z=\left\{z_{i} \mid i \in I\right\}^{1}$ of $\mathcal{D}$ in a dynamics $\mathcal{D} / k<z>$. An element $\xi$ in $\mathcal{D}$ is said to be observable with respect to $z$ if it is algebraic ${ }^{2}$ over $k<z>$.

For each configuration of the switching system (6), $\dot{\xi}=$ $f_{i}(\xi, u), i \in Q$, an algebraic function satisfying $\xi=$ $\varpi\left(z, \dot{z}, \ldots, z^{(n)}\right), n<\infty$ can be found (computations are not reported here for the sake of place). According to Lemma 1, it can be concluded that, for each configuration, the system (6) is observable.

Lemma 1 means in other words that observability is equivalent to the following fact: any system variable, a state variable for instance, can be expressed as a function of the input and output variables and of their time derivatives up to some finite order. Thus, if one is able to estimate the successive time derivatives of the output, it is possible to reconstruct the state of the system. In the next section, a new type of numerical differentiator, based on differential algebra concepts and already developed in [9] and [14], is briefly recalled.

\subsection{Algebraic Numerical Differentiation}

Let $y(t)=x(t)+n(t)$ be a noisy measurement on a finite time interval of a real-valued analytic signal $x(t)$ defined by the convergent power series $x(t)=\sum_{i>0}^{\infty} x^{(i)}(0) \frac{t^{i}}{i !}$, where $0 \leq t<\rho$. The objective is to estimate the successive time derivatives of $x(t)$ using the noisy measurement $y(t)$.

\footnotetext{
1 Usually, $z$ is the set $\{u, y\}$ of input and output variables and $\xi$ is the set $x$ of state variables.

$2 \xi$ can be expressed as an algebraic function of the components of $z$ and a finite number of their time derivatives.
} 
For this, approximate $x(t)$ in the interval $(0, \varepsilon), 0<\varepsilon \leq \rho$ by its truncated Taylor expansion $x_{N}(t)=\sum_{i>0}^{N} x^{(i)}(0) \frac{t^{i}}{i !}$. It reads in the operational domain as

$$
s^{N+1} x_{N}(s)=s^{N} x_{N}(0)+s^{N-1} \dot{x}_{N}(0)+\ldots+x_{N}^{(N)}(0)
$$

In order to obtain an estimate of the $n$-th order time derivative of $x(t)$, one can annihilate the remaining coefficients $x_{N}^{j}(0), j \neq n$ by multiplying by linear differential operators of the form

$$
\Pi_{\kappa}^{N, n}=\frac{d^{n+\kappa}}{d s^{n+\kappa}} \cdot \frac{1}{s} \cdot \frac{d^{N-n}}{d s^{N-n}} \quad \kappa \geq 0
$$

It yields the following estimate for $x^{(n)}(0)$

$$
\frac{\tilde{x}_{N}^{(n)}(0)}{s^{\nu+n+\kappa+1}}=\frac{(-1)^{n+\kappa}}{(n+\kappa) !(N-n) !} \frac{1}{s^{\nu}} \Pi_{\kappa}^{N, n}\left(s^{N+1} \hat{x}\right)
$$

which is strictly proper whenever $\nu$ is of the form $\nu=N+$ $1+\mu, \mu \geq 0$. After transferring both equations (9) into the time domain, and several calculations, the estimate of the $n^{t h}$-order derivative of $x(t)$ takes the following form ${ }^{3}$

$$
\begin{aligned}
\tilde{x}_{N}^{(n)}(0)= & A(\nu, n, \kappa, N) \sum_{i=0}^{N-n} B(i, n, N) \sum_{j=j_{0}}^{n+\kappa} C(i, j, n, \kappa) \\
& \int_{0}^{1}(1-\tau)^{\nu+\kappa-i-j-1}(-\tau)^{i+j} x(T \tau) d \tau
\end{aligned}
$$

where $j_{0}=\max (0, k-i)$ and

$$
\begin{aligned}
& A(\nu, n, \kappa, N)=\frac{(-1)^{n+\kappa}(\nu+n+\kappa) !}{(n+\kappa) !(N-n) !} \\
& B(i, n, N)=\left(\begin{array}{l}
N-n \\
i
\end{array}\right) \frac{(N+1) !}{(n+i+1) !} \\
& C(i, j, n, \kappa)=\left(\begin{array}{l}
n+\kappa \\
j
\end{array}\right) \frac{(n+i) !}{(i+j-\kappa) !}
\end{aligned}
$$

Remark 2. An $n^{\text {th }}$-order truncated Taylor expansion is appropriate for estimating the $n^{t h}$-order derivative ${ }^{4}$.

The estimator only contains the integrations, with lowpass filter properties, which attenuates the effects of the noises.

\subsection{Discrete State Identification Design}

In this section, a scheme for the discrete state identification is given, which includes two parts, one based on the kinematic constraint and the other based on the dynamic constraint.

Kinematic Constraint For the ideal case and slipping case, the nonholonomic constraint is satisfied, so

$$
\mathcal{C}_{k}=\dot{x} \cos \theta+\dot{y} \sin \theta=0
$$

One has the knowledge of $\mathcal{C}_{k}$ as follows:

$$
\mathcal{C}_{k}=\tilde{\dot{x}} \cos \theta+\tilde{\dot{y}} \sin \theta
$$

where $\tilde{\dot{x}}$ and $\tilde{\dot{y}}$ are estimates of $\dot{x}$ and $\dot{y}$ obtained through algebraic numerical differentiation.

3 See [14] for details.

4 See Lemma 1 in [14].

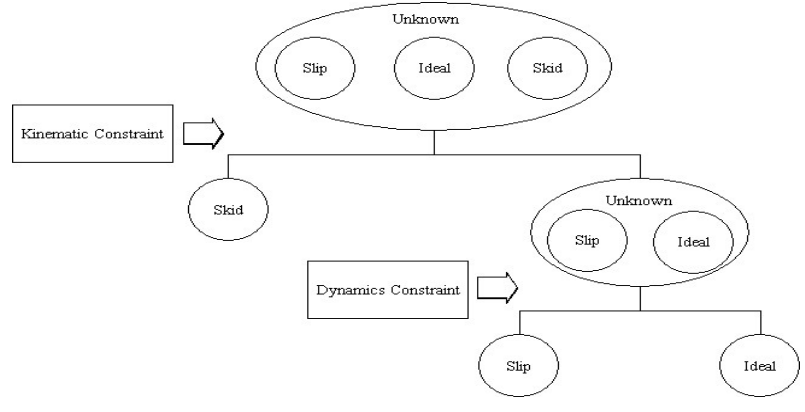

Fig. 2. Discrete State Identification Scheme

If $\mathcal{C}_{k} \neq 0$, the real tire/road contact condition is skidding and thus $q=1$. If $\mathcal{C}_{k}=0$, one has to distinguish the ideal and slipping cases. This is done as follows.

Dynamic Constraint Compare the system for the ideal case and the one for the slipping case, one has a dynamics constraint, which can be written as

$$
\mathcal{C}_{d}=\dot{v}_{y}+\frac{r^{2}}{3 I_{\phi}}\left(u_{1}+u_{2}\right)
$$

In systems (3) and (5), one has:

Thus:

$$
v_{y}=\dot{y} \cos \theta-\dot{x} \sin \theta
$$

$$
\dot{v}_{y}=\ddot{y} \cos \theta-\ddot{x} \sin \theta-\dot{\theta}(\dot{x} \cos \theta+\dot{y} \sin \theta)
$$

and the knowledge of $\dot{v}_{y}$ (and so of $\mathcal{C}_{d}$ ) is obtained using algebraic numerical differentiators. Then, one knows if (6) switches to the ideal case $\left(\mathcal{C}_{d}=0\right)$ and $q=0$ or to the slipping case $\left(\mathcal{C}_{d} \neq 0\right)$ and $q=2$.

\section{ESTIMATION OF UNCERTAIN PARAMETERS}

In practice, some parameters in these models are difficult to be known, and may change when perturbations arise, such as loading or unloading "passengers", weather changing, abrasion of the tire. So, it is necessary to build an estimator of those uncertain parameters for each configuration of the system (6). In the given scheme, the only parameter that must be identify is $\alpha=\frac{r^{2}}{3 I_{\phi}}$, which appears in equation (13).

\subsection{Ideal case}

From the kinematic part, one obtains:

$$
\begin{aligned}
& \tilde{\dot{v}}_{y}=-\ddot{x} \sin \theta+\ddot{y} \cos \theta \\
& \tilde{\dot{v}}_{\theta}=l \ddot{\theta}
\end{aligned}
$$

where $\tilde{\dot{v}}_{y}, \tilde{\dot{v}}_{\theta}$ represent the estimates of $\dot{v}_{y}, \dot{v}_{\theta}$, respectively. Then, we turn to the dynamical part and the parameter $\alpha=\frac{r^{2}}{3 I_{\phi}}$ can be written as:

$$
\tilde{\alpha}= \begin{cases}-\frac{\tilde{\dot{v}}_{y}}{u_{1}+u_{2}} & \text { if } u_{1}+u_{2} \neq 0 \\ -\frac{\tilde{v}_{\theta}}{u_{1}-u_{2}} & \text { if } u_{1}-u_{2} \neq 0\end{cases}
$$

If the inputs are zero, it is not required to estimate this parameter. 


\subsection{Skidding case}

The following relations can be obtained from the kinematic part of (4):

$$
\begin{aligned}
& \tilde{v}_{x}=\dot{x} \cos \theta+\dot{y} \sin \theta \\
& \tilde{v}_{y}=-\dot{x} \sin \theta+\dot{y} \cos \theta \\
& \tilde{v}_{\theta}=l \dot{\theta} \\
& \tilde{\dot{v}}_{x}=\ddot{x} \cos \theta-\dot{x} \sin \theta \dot{\theta}+\ddot{y} \sin \theta+\dot{y} \cos \theta \dot{\theta} \\
& \tilde{\dot{v}}_{y}=-\ddot{x} \sin \theta+\ddot{y} \cos \theta-\tilde{v}_{x} \dot{\theta} \\
& \tilde{\dot{v}}_{\theta}=l \ddot{\theta}
\end{aligned}
$$

After some calculations, one obtains:

$$
\tilde{\alpha}=\left\{\begin{array}{cl}
-\frac{\tilde{\dot{v}}_{y}+\frac{\tilde{v}_{x} \tilde{v}_{\theta}}{3 l}}{u_{1}+u_{2}} & \text { if } u_{1}+u_{2} \neq 0 \\
-\frac{\dot{\hat{v}}_{\theta}}{u_{1}-u_{2}} & \text { if } u_{1}-u_{2} \neq 0
\end{array}\right.
$$

Remark 3. The parameters $G_{x}, G_{y}$ and $I_{\theta}$ are not estimated because they are not used in the identification scheme of the discrete state.

\section{SIMULATION RESULTS}

The simulations are carried out for three scenarii to consider all the possibilities: acceleration, uniform motion and deceleration. For the sake of place, only the simulation results for the acceleration case are reported here. The physical parameters used in the simulation are: $G_{x}=$ $0.4, G_{y}=1, \epsilon=10^{-4}, I_{\theta}=500 \mathrm{Kg} \cdot \mathrm{m}^{2}, I_{\phi}=1.6 \mathrm{Kg} \cdot \mathrm{m}^{2}, l=$ $1 m, r=0.35 m, m=1000 K g$, and normal Gaussian noises with variance $\delta^{2}=0.1$ are added to the measurements $s=(x, y, \theta)^{T}$.

\subsection{State Identification for WMR}

This simulation mainly focuses on the identification of the discrete state $q$, which represents the tire/road condition: ideal $(q=0)$, skidding $(q=1)$ or slipping $(q=2)$. The acceleration procedure of the simulation encompasses 6 different successive conditions: ideal-skidding-slippingskidding-ideal-slipping, and is shown in Fig. 4 in dashed blue line. It is worth mentioning that accelerating just means acceleration on $v_{y}$, not on $v_{\theta}$. The Top-Left (TL) of Fig. 3 shows the trajectory of the mobile robot where the red dashed lines is the ideal case, the blue dashed lines correspond to the skidding phenomenon, and the green dashed lines to the slipping phenomenon. The other three graphs of Fig. 3 gives the behaviour of the velocities $v_{y}, v_{x}$, and $v_{\theta}$. The estimates of $\tilde{v}_{y}, \tilde{v}_{x}$, and $\tilde{v}_{\theta}$, built with algebraic numerical differentiation, are given in solid lines and the real values are in dashed lines. In Fig. 4, one can see the identification of the tire/road contact condition. Due to the noise measurements in the simulations, a threshold was introduced on the fulfilment of the constraints.

\subsection{Uncertain Parameter Estimation}

In order to evaluate the efficiency of the estimation of the uncertain parameters, only acceleration case with constant tire/road condition is considered in this simulation. First,
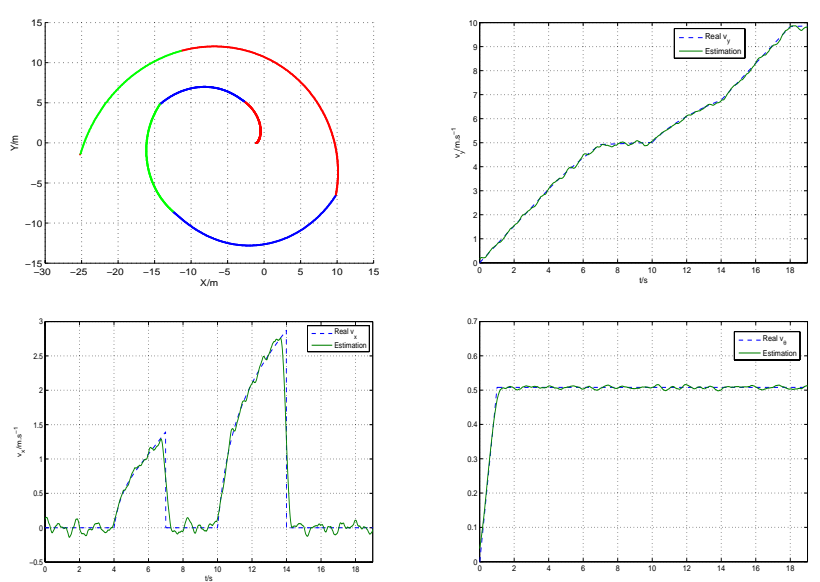

Fig. 3. (TL)Trajectory of the WMR; (TR)The longitude velocity $v_{y}$; (BL)The lateral velocity $v_{x} ;(\mathrm{BR})$ The angle velocity $v_{\theta}$.

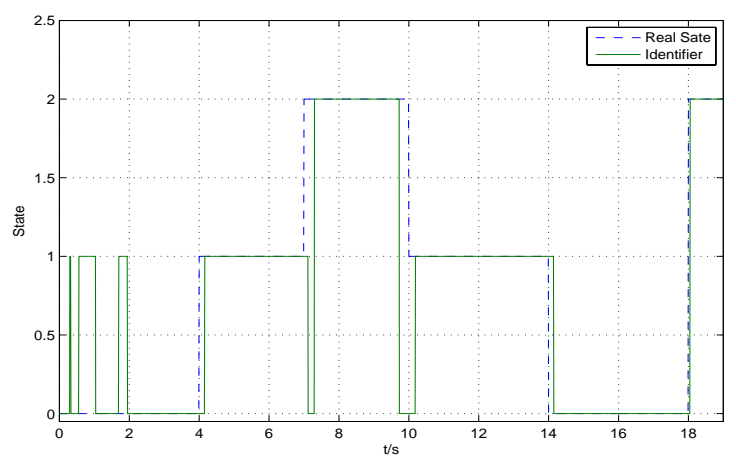

Fig. 4. Identified discrete state.

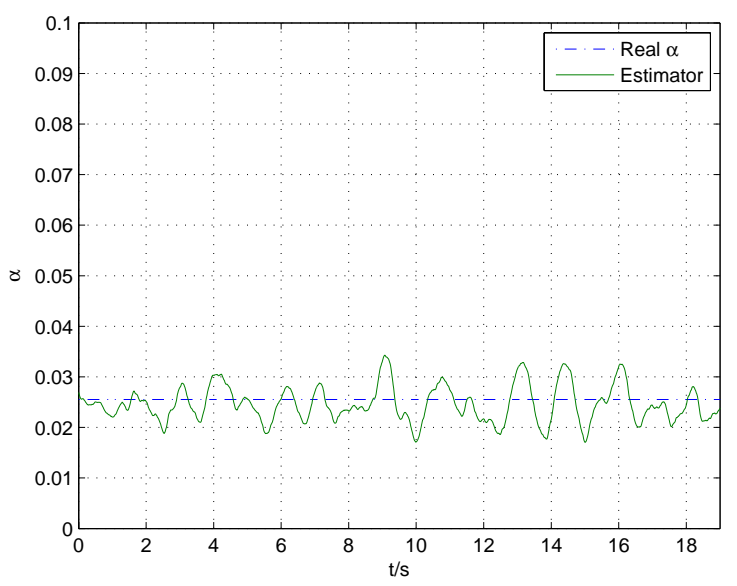

Fig. 5. Unknown Parameter Estimation for Ideal Condition.

the tire/road contact condition is configured as the ideal condition. The estimate of $\alpha$ is given in Fig. 5. Then, the tire/road contact condition is configured as the skidding condition. In Fig. 6 is given the estimate of $\alpha$. 


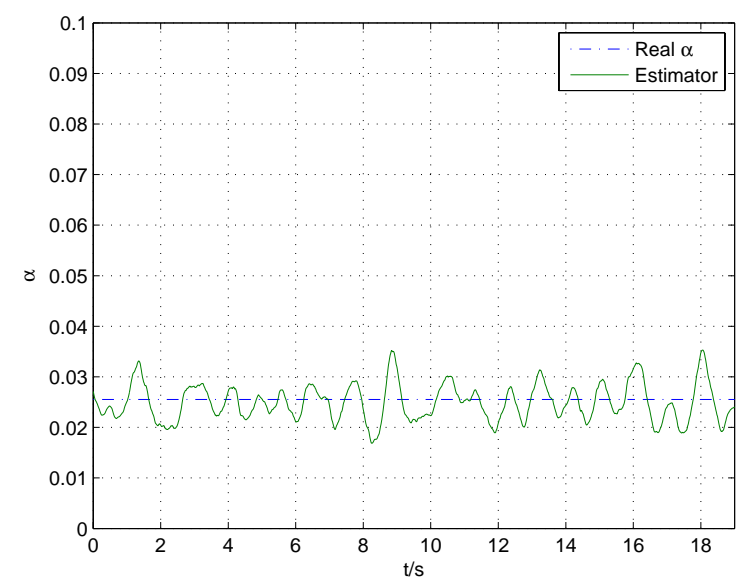

Fig. 6. Unknown Parameter Estimation for Skidding Condition.

\section{CONCLUSION}

In this paper, an identification scheme for the tire/road contact condition has been introduced. This identification scheme is based on algebraic numerical differentiation. Using the kinematic and dynamic constraints, the discrete states of a switched system, corresponding to tire/road contact conditions, has been identified. Meanwhile, considering that the parameters in the system are not known, estimators for these parameters have also been built. Measurement noises have been considered to make the simulation more realistic.

\section{REFERENCES}

[1] J.-P. Barbot, M. Fliess, T. Floquet. An algebraic framework for the design of nonlinear observers with unknown inputs. $46^{\text {th }}$ IEEE Conference on Decision and Control, New-Orleans, USA, 2007.

[2] C. Canudas-de-Wit, M. L. Petersen, A. Shiriaev. A New Nonlinear Observer for Tire/Road Distributed Contact Friction. Conference on Decision and Control, 2003.

[3] C. Canudas de Wit, O. Sordalen. Exponential stabilization of mobile robots with nonholonomic constraints. IEEE Trans. on Automatic Control, 1992, 37, (11), pp. 1791-1797.

[4] B. D'Andréa-Novel, G. Bastin, G. Campion. Modelling and Control of Non Holonomic Wheeled Mobile Robots. IEEE international Conference on Robotics and Automation, 1991.

[5] M. Defoort, J. Palos, A. Kökösy, T. Floquet, W. Perruquetti, D. Boulinguez. Experimental motion planning and control for an autonomous nonholonomic mobile robot. IEEE International Conference on Robotics and Automation, Roma, Italy, 2007.

[6] M. Defoort, T. Floquet, A. Kökösy, W. Perruquetti. Sliding Mode Formation Control for Cooperative Autonomous Mobile Robots. IEEE Transactions on Industrial Electronics, Vol. 55, no. 11, pages 39443953, 2008.

[7] S. Diop, M. Fliess. Nonlinear Observability, Identifiability, and Persistent Trajectories. Proceedings of the 30th Conference on Decision and Control, 1991.
[8] M. Ellouze, B.d'Andréa-Novel. Modeling and control of unicycle type-robot in presence of decoupled slipping and skidding effects. European Control Conference ECC'99, 1999.

[9] M. Fliess, C. Join, H. Sira-Ramírez. Nonlinear Estimation is easy. Int. J. Modelling Identification Control, Vol. 4, pages 12-27, 2008.

[10] M. Fliess, S. Fuchshumer, M. Schöberl, K. Schlacher, H. Sira-Ramirez. An introduction to algebraic discrete-time linear parametric identification with a concrete application. Journal Européen des Systèmes Automatisés (JESA) 42, 2-3 (2008) 210-232.

[11] W. Leroquais and B. D'Andréa-Novel. Modelling and control of wheeled mobile robots not satisfying ideal velocity constraints-the unicycle case. Proceedings of the $35^{\text {th }}$, Conference on Decision and Control, Kobe, Japan, December 1996.

[12] W.-S. Lin, L.-H. Chang, P.-C. Yang. Adaptive Critic Anti-Slip Control of Wheeled Autonomous Robot. IET Control Theory Application, Vol.1, No.1, pages 51-57 January 2007.

[13] C. B. Low, D. Wang. GPS-Based Path Following Control for a Car-Like Wheeled Mobile Robot With Skidding and Slipping. IEEE Transactions on Control Systems Technology, Vol. 16, No. 2, pages 340-347, March 2008.

[14] M. Mboup, C. Join, M. Fliess. A revised look at numerical differentiation with an application to nonlinear feedback control. Proc. 15th Medit. Conf. Control Automat., Athens, 2007.

[15] C. Prieur, A. Astolfi. Robust stabilization of chained systems via hybrid control. IEEE Trans. Automat. Control, Vol. 48, pages 1768-1772, 2003.

[16] N. K. M'Sirdi, A. Rabhi, L. Fridman, J. Davila, Y. Delanne. Second order sliding-mode observer for estimation of vehicle dynamic parameters. International Journal of Vehicle Design, Vol. 48, No. 3/4, pages 190-207, 2008.

[17] J. Villagra, B. D'Andréa-Novel, M. Fliess, H. Mounier. Estimation of longitudinal and lateral vehicle velocities: an algebraic approach. American Control Conference, 2008.

[18] C. C. Ward, K. Iagnemma. Model-Based Wheel Slip Detection for Outdoor Mobile Robots. IEEE International Conference on Robotics and Automation, Roma, Italy, 2007. 